\title{
Neurons excited to death by SLE autoantibodies
}

B $y$ holding a gated ion channel in an open position, autoantibodies found in $40-50 \%$ of patients with systemic lupus erythematosus (SLE) alter synaptic transmission and cause neurotoxicity. This mechanism, according to new data obtained using mouse hippocampal slices and published in Proceedings of the National Academy of Sciences, might underlie the neuropsychiatric manifestations of SLE.

Autoantibodies that bind doublestranded DNA (dsDNA) are a diagnostic criterion for SLE. Disease symptoms, however, generally arise as a result of cross-reactivity of these antibodies with other self-antigens. One such target is the $N$-methyl $D$-aspartate receptor (NMDAR), through which the flow of cations is controlled by voltage and binding of glutamate and glycine. Previously, Patricio Huerta-a lead author of the new reportand colleagues showed that NMDARreactive autoantibodies, which have been detected in up to $50 \%$ of patients with SLE, cause neuronal damage and impair cognition and behavior in mice. This disruption is dependent on a breach of the blood-brain barrier, as a result, for example, of immune challenge.

Pyramidal neurons of the hippocampus, which express the NMDAR subunits that are bound by the SLE autoantibodies, were known to be a preferred target for the neurotoxicity. The researchers therefore chose to use hippocampal slices, commonly used in electrophysiological studies, to investigate the neurotoxic mechanism. In vivo, compartmentalization of hippocampal neurons and downstream signaling controls different functions. "This intricate structural-functional distribution of the NMDARs cannot be reproduced in cell expression systems," explains Huerta, "but it might be a crucial feature in how lupus autoantibodies ultimately exert their neurotoxic effects." Using the hippocampal slices, the investigators recorded postsynaptic membrane potentials and currents,
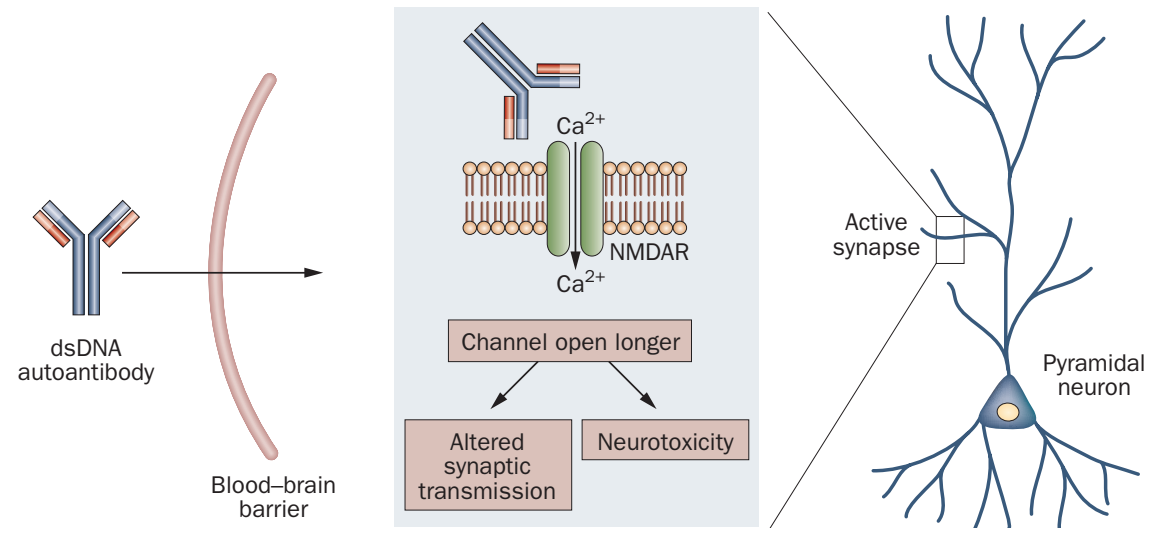

pharmacologically isolated the NMDARmediated responses, and used a liveimaging method to monitor mitochondrial permeability. An irreversible transition in mitochondrial membrane permeability (mPT) marks the point at which altered synaptic responses turn into NMDARmediated excitotoxicity.

"The most important finding was that the NMDAR-reactive autoantibody acted as a positive modulator of NMDARmediated synaptic responses and toxicity, rather than as a direct agonist", says Huerta. The antibodies preferentially bound to the open NMDAR pore and functioned only on neurons with active synapses. This suggests that the binding prolongs the open state, which would increase the influx of calcium ions. Receptor-mediated changes in intracellular calcium concentration control gene transcription responses, and toxic levels cause the mPT.

The effect is dose-dependent, with modulated transmission resulting in neurotoxicity at higher doses of autoantibody. "This mirrors what occurs in patients," says Huerta, "in a subset of whom neuropsychiatric symptoms are observed to be reversible, whereas after several iterations of neuropsychiatric episodes, or an antibody insult of high concentration, a greater loss of neurons may result in permanent cognitive disturbance."
So what does it mean for the understanding and treatment of neuropsychiatric SLE? The condition is becoming more common, as improved treatments and survival rates for SLE provide time for the emergence of brain pathology. Serum levels of NMDAR-crossreactive autoantibodies do not correlate with neuropsychiatric symptoms, but this can be explained by their restricted access to cerebrospinal fluid. Huerta and colleagues are interested in the conditions that lead to antibody entry to the brain, and also plan to use hippocampal-dependent behavioral assays in mice to reinforce the link between synaptic alterations and behavioral responses.

The work does have potential clinical applications, says Dimitrios Boumpas, an expert in neuropsychiatric SLE, but first it must be established "how relevant this effect may be for human lupus, and how important compared to other mechanisms that cause neuropsychiatric symptoms."

Emma Leah

Original article Faust, T. W. et al. Neurotoxic lupus autoantibodies alter brain function through two distinct mechanisms. Proc. Natl Acad. Sci. USA 107, 18569-18574 (2010)

Further reading Bertsias, G. K. \& Boumpas, D. T. Pathogenesis, diagnosis and management of neuropsychiatric SLE manifestations. Nat. Rev. Rheumatol. 6, 358-367 (2010) 